\title{
Existence and uniqueness of solution for multidimensional parabolic PDAEs arising in semiconductor modeling
}

\author{
Giuseppe Ali $^{1}$ and Nella Rotundo ${ }^{2}$ \\ ${ }^{1}$ University of Calabria \\ ${ }^{2}$ Weierstrass Institute for Applied Analysis and Stochastics
}

May 5, 2020

\begin{abstract}
We consider a linear RLC network, modeled by differential-algebraic equations, containing distributed semiconductor devices, modeled by multi-dimensional parabolic-elliptic equations. With appropriate coupling conditions, we obtain a system of multidimensional partial differential-algebraic equations. For the resulting system, we prove an existence and uniqueness result, and study the asymptotic behavior of the solutions.
\end{abstract}

\section{Hosted file}

Ali-Rotundo-Submission.pdf available at https://authorea.com/users/292907/articles/420756existence-and-uniqueness-of-solution-for-multidimensional-parabolic-pdaes-arising-insemiconductor-modeling 